We are well aware of the many problems faced by those in charge of an acute children's ward today, and that wards whose design is satisfactory on other grounds will be in use for a long time. Nevertheless, without asking too much of busy resident doctors and ward sisters, we believe that the knowledge of the facts and the applications of the conclusions of this study could do much to improve the situation.

We are indebted to the M.R.C. for continuing support. We are also grateful to the medical and nursing staff of all the hospitals who showed the patient and good-tempered co-operation without which this type of study would be impossible.

\section{References}

Ferris, J. A. J., Aherne, W. A., Locke, W. S., McQuillin, J., and Gardner, P. S., (1973). British Medical fournal, 2, 439.

Brocklebank, T., Court, S. D. M., McQuillin, J., and Gardner, P. S. (1972). Lancet, 2, 497.

Ditchburn, R. K., McQuillin, J., Gardner, P. S., and Court, S. D. M. (1971) British Medical Fournal, 3, 671.

Gardner, P. S., McQuillin, J., and McGuckin, R. (1970). Fournal of Hygiene, $68,575$.

Gardner, P. S., Stanfield, J. P., Wright, A. E., Court, S. D. M., and Green, C. A. (1960). British Medical fournal, 1, 1077.

Gardner, P. S., et al. (1967). British Medical fournal, 4, 316.

McQuillin, J., and Gardner, P. S. (1968). British Medical fournal, 1, 602.

Sterner, G. (1972). British Medical fournal, 1, 51.

Sturdy, P. M., McQuillin, J., and Gardner, P. S. (1969). Fournal of Hygiene, 67,659 .

\title{
Excretion Urography in Acute Renal Failure
}

\author{
W. R. CATTELL， C. S. MCINTOSH， I. F. MOSELEY， I. KELSEY FRY
}

British Medical fournal, 1973, 2, 275-278

\section{Summary}

High-dose excretion urography has been carried out in 32 patients presenting with non-obstructive acute oliguric or non-oliguric renal failure. An early, dense, persisting nephrogram has been observed in all patients with acute uncomplicated tubular necrosis and in patients with acute oliguric pyelonephritis. This appearance is modified by the presence of pre-existing renal disease. Different patterns have been observed in patients with acute glomerular disease, severe renal ischaemia, and chronic glomerular disease. The study demonstrates that careful analysis of the evolution of the nephrogram in patients with acute renal failure provides valuable information as to the nature of the parenchymal disease.

\section{Introduction}

High-dose excretion urography is of established value in the investigation of patients with non-oliguric renal failure, both to find or exclude treatable postrenal obstruction and, by defining the renal outlines, to help diagnose the nature and severity of chronic parenchymal disease (Fry and Cattell, 1971a, 1971b). It has been less extensively used in patients with oliguric renal failure, partly because of fear that the investigation might be hazardous (Schencker, 1964; Fry and Cattell, 1970) and partly because of doubt whether any useful information could be obtained. Recent studies of our own and others (Mahaffy et al., 1969; Brown et al., 1970; Meadows et al., 1971) have, however, shown that high-dose urography is safe in oliguric subjects and is capable of defining both renal size and the presence or absence of obstruction.

In the course of these studies it became apparent that critical examination of changes in the density of the kidney during

\footnotetext{
St. Bartholomew's/St. Leonard's Regional Renal Unit ar' the Departments of Radiology, St. Bartholomew's Hospital, Loudon EC1A 7BE, and St. Leonard's Hospital, London N.1

W. R. CATTELL, M.D., F.R.C.P., Consultant Nephrologist

C. S. MCINTOSH, M.B., M.R.C.P., Senior Medical Registrar

(Present address: Westminster Hospital, London S.W.1)

I. F. MOSELEY, M.B., M.R.C.P., Senior Registrar in Diagnostic Radiology (Present address: Mount Zion Hospital and Medical Centre, San

I. KELSEY FRY, D.M., F.R.C.P., Consultant Radiologist
}

excretion urography-the nephrographic pattern (Fry and Cattell, 1972)-could also yield information about the nature of the parenchymal disease in patients with acute non-obstructive renal failure (Moseley et al., 1971). To extend these observations we now report the urographic findings in 32 patients presenting with acute non-obstructive oliguric and nonoliguric renal failure. This study clearly indicates that careful analysis of the nephrographic pattern taken in conjunction with renal size and the presence or absence of pelvicalyceal filling will provide valuable information about the nature of the renal lesion in these patients.

\section{Patients and Methods}

Altogether, 32 patients, aged 22-69 years, have been studied (see table*). All had presented with renal failure of recent acute onset without any previous history of renal disease. In many cases a presumptive clinical diagnosis had been made but in all some doubt existed as to the true diagnosis. Most (22) were oliguric (less than $500 \mathrm{ml}$ of urine per day) and all were uraemic. Prerenal circulatory failure had been excluded by the time of study as judged by persisting oliguria and/or increasing uraemia despite adequate volume replacement and intensive diuretic treatment with mannitol and frusemide. For the purpose of the present investigation patients with postrenal obstruction were also excluded.

High-dose excretion urography was performed as soon as possible after admission to hospital and exclusion of prerenal circulatory failure. Severely uraemic and fluid-overloaded patients were dialysed to improve their clinical condition before the investigation.

Urographic Technique.-Fluid restriction was avoided. $1 \mathrm{ml} / \mathrm{lb}$ body weight $(2 \cdot 2 \mathrm{ml} / \mathrm{kg}$ ) Hypaque $45 \%$ (sodium diatrizoate) or an equivalent dose of Conray 420 (sodium iothalamate) was injected intravenously over a period of 3-5 minutes. Preliminary tomograms of the renal areas were obtained at the same time as the control plain films. Whenever possible films were taken immediately at the end of injection, at $5,10,30$, and 60 minutes, and at intervals up to 24 hours. Tomograms were always obtained immediately after injection and with most of the subsequent films. In a few patients the full range of films was not taken owing to their clinical condition. Special care was taken to ensure that tomographic cuts throughout the examination were comparable with regard both to radiographic exposure and to level of cut.

*Further clinical details are available on request to: Dr. W. R. Cattell, Department of Nephrology, St. Bartholomew's Hospital, London EC1A 7BE 
Clinical and Radiological Findings in the 32 Patients Studied

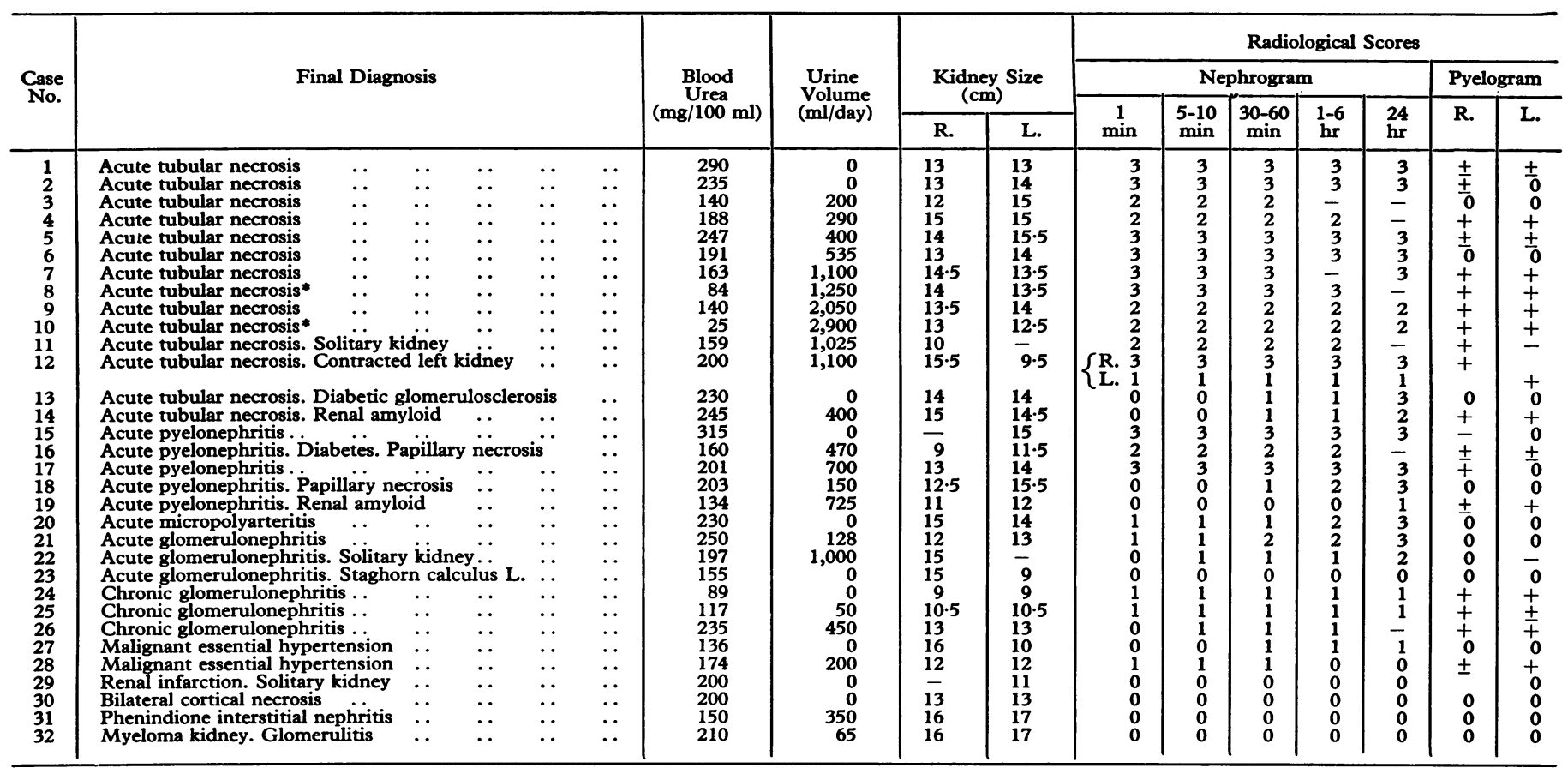

*Patients studied in the early diuretic recovery phase.

Assessment of Films.-The films were examined with special reference to kidney size, nephrogram, and the presence or absence of a pyelogram. With respect to the nephrogram the following features were noted: time of first appearance, maximum density observed, change in density during the investigation, and total duration. The density of the nephrogram was scored on a scale of 1-3 as follows. A score of 1 represented a nephrogram which was just discernible and 3 represented a nephrogram as dense as would be expected immediately after injection in a patient with normal renal function given a large dose of contrast medium. A score of 2 was given to a nephrogram of intermediate density. The pyelogram was recorded as absent (0) or present $(+)$. Faint traces of calyceal filling insufficient to show any calyceal anatomy were separately recorded $( \pm)$.

Normal Nephrographic Pattern.-For comparison, the pattern of development of the nephrogram and the pyelogram were studied in the same way (but without tomography) in 12 patients in whom excretion urography had been requested as part of their hospital investigation. In all renal function was subsequently shown to be normal and there was no anatomical abnormality of the urinary tract.

Diagnosis. - A histological diagnosis was obtained in 19 of 32 patients. The final diagnosis in each patient is given in the table. The absence of histological confirmation in the remainder is due to the fact that we considered it unjustifiable to carry out renal biopsy in patients with acute tubular necrosis or suppurative pyelonephritis unless the clinical course was complicated or the diagnosis in serious doubt. In these cases the final clinical diagnosis of acute tubular necrosis was based on reversible acute renal failure developing in a clinical setting of circulatory failure and hypotension with or without tissue injury. In all instances exogenous nephrotoxins, prerenal or extrarenal factors, and obstruction had been excluded. The clinical diagnosis of suppurative pyelonephritis was based on the presence of fever, loin pain, bacteriuria, and improvement after antibacterial therapy.

\section{Results}

Kidney Size and Outline.-The renal outline was clearly defined in all save one patient. The exception (case 11) was a man who had undergone partial nephrectomy for a hypernephroma in a solitary kidney. In this patient, probably as a result of the recent surgery, the upper part of the kidney could not be defined. Unequivocal bilateral reduction in renal size was observed in only two patients, both of whom had chronic glomerulonephritis. Unilateral small kidneys were observed in four patients, two of whom had "surgical disease" of the small kidneys (previous operation in one and calculous disease in the other). One had acute pyelonephritis with papillary necrosis while the fourth had malignant hypertension. In the remainder, on average, the kidney size was in the upper normal range.

Nephrogram and Pyelogram.-The radiological findings are set out in the table.

Control Subjects.-An obvious nephrogram was seen in all 12 patients. It was present and maximal at the end of injection of contrast medium and began to fade during the next 30 minutes. A faint nephrogram was seen at six hours in six patients and in one a very faint nephrogram was seen at 24 hours. A dense pyelogram was always shown, most dense between 10 and 30 minutes after injection.

Acute Tubular Necrosis.-There were 14 patients in whom a final diagnosis of acute renal failure due to acute tubular necrosis was made. In 10 there was no associated renal disease. In one of the remaining four patients (case 11) acute tubular necrosis was sustained during partial nephrectomy for hypernephroma in a solitary kidney. Case 12 was known to have had a calculus removed from the left kidney some years previously and this kidney was reduced in size. The remaining two patients (cases 13 and 14) both had acute tubular necrosis complicating preexisting bilateral renal disease. In 12 of the 14 cases a distinctive nephrographic pattern was observed. All had an obvious nephrogram (grade 2 or 3 ) at the end of the injection and this nephrogram persisted without change in density up to at least 60 minutes and was still evident and unchanged in all patients in whom films were taken up to 24 hours. In all save one the nephrographic appearances were similar on the two sides. The exception (case 12) had a dense persisting nephrogram on the right side but only a faint persisting nephrogram on the left -a kidney known to be the site of previous surgery. An early, persisting nephrogram was not observed in the two patients with acute tubular necrosis and severe pre-existing renal damage (cases 13 and 14) in both of whom no nephrogram was 
seen until 30 minutes, after which it increased in density up to 24 hours. No pyelogram or only a very faint pyelogram was observed in six of the 14 patients. With one exception the development of a pyelogram in patients with uncomplicated acute tubular necrosis appeared to relate to the presence or absence of oliguria. Thus, a pyelogram was observed in all patients with daily urine outputs in excess of one litre. The exception (case 4) had a ruptured aortic aneurysm. A definite pyelogram was seen in this case in spite of persisting oliguria up to the time of his death one week after excretion urography.

Acute Pyelonephritis. - Three of the five patients in whom acute renal failure was associated with severe pyelonephritis showed the same nephrographic pattern as uncomplicated acute tubular necrosis-all had an early grade 2 or 3 persisting nephrogram. A definite pyelogram was seen on one side in the only non-oliguric patient of the three. A different nephrographic pattern was observed in the remaining two patients (cases 18 and 19). Neither showed a nephrogram until 30 minutes. In one case the nephrogram then became increasingly dense up to 24 hours and was not associated with a pyelogram. This patient had Gram-negative septicaemia and at necropsy was found to have severe papillary necrosis and severe suppurative pyelonephritis. The other atypical case had severe glomerular disease due to amyloid deposition in addition to the acute pyelonephritis. On excretion urography only a faint (grade 1) nephrogram was observed which did not appear for 24 hours though there was a definite pyelogram on one side.

Acute Glomerulonephritis.-There were four patients with acute glomerulitis, one of whom had micropolyarteritis. Three of the four showed slowly developing but increasingly dense nephrograms and no pyelogram. The fourth, a patient with severe anuric proliferative glomerulonephritis, showed no nephrogram and no pyelogram at any stage.

Chronic Glomerulonephritis.-The three patients presenting with acute renal failure subsequently shown to be due to chronic glomerulonephritis all had similar urographic findingsan early, faint nephrogram which persisted unchanged up to 24 hours accompanied by a pyelogram.

Malignant Essential Hypertension.-There were two patients with malignant essential hypertension. One showed a faint early nephrogram which disappeared at one hour and was associated with a pyelogram. The other, who was anuric, showed a delayed faint but persisting nephrogram and no pyelogram.

Miscellaneous.-Finally, there were four patients with differing diseases in whom the same urographic findings were observed-no nephrogram or pyelogram at any stage up to 24 hours. One patient had total kidney infarction as a result of prolonged renal artery occlusion during surgery to a solitary kidney, one with severe pancreatitis had bilateral cortical necrosis, and one had acute interstitial nephritis complicating phenindione hypersensitivity. Biopsy in the latter case showed gross interstitial oedema and almost complete loss of tubular lumen possibly due to tubular compresison. The glomeruli in this patient appeared entirely normal. The other patient, a young man of 34, had fulminating myelomatosis undiagnosed before excretion urography. Subsequent renal biopsy showed extensive tubular blockage with protein casts and in addition, unexpectedly, glomerular changes of proliferative glomerulonephritis.

Toxic Effects. - Careful prospective study failed to show any adverse effects attributable to excretion urography.

\section{Discussion}

With the advent of effective dialysis therapy, powerful broadspectrum antibiotics, and provision for intensive nursing care few patients should now die of acute, potentially reversible renal failure. Such an approach demands skilled medical and nursing care, intensive and often prolonged therapy, and the use of expensive drugs and equipment. For the most effective use of these resources it is increasingly important to establish the nature of the underlying disease so that correct decisions may be taken with respect to the immediate and long-term management of the patient presenting for the first time with uraemia (Wrong, 1971). In many patients presenting with acute renal failure a reasonably accurate diagnosis can be made from a careful history and physical examination, but in others considerable doubt remains as to the precise nature of the underlying disease. To resolve this problem many special investigations have been recommended, including examination of the urinary sediment (Levinsky, 1966), measurement of urine/plasma urea ratio (Perlmutter et al., 1959; Eliahou, 1964; Luke and Kennedy, 1967), urine/plasma osmolar ratio (Eliahou and Bata, 1965), and urinary sodium concentration (Graber and Sevitt, 1959; Levinsky, 1966; Platts, 1966). While sometimes diagnostic, all too often the results of these investigations do not clearly separate prerenal circulatory failure from acute intrinsic renal disease or chronic renal disease. Nor do they give any information about the nature of acute intrarenal damage (Luke and Kennedy, 1967; Rosenbaum, 1967). Transcutaneous renal biopsy will in most cases provide a histological diagnosis in patients with acute renal failure. It is, however, an investigation which should not be undertaken lightly in such patients because of the increased hazard of haemorrhage from the puncture site.

In the course of an earlier study of patients with oliguric renal failure (Brown et al., 1970) it was noted that opacification of the kidney with contrast medium followed different patterns which appeared to correlate with the nature of the parenchymal disease (Moseley et al., 1971). The present study bears out these preliminary observations.

Patients with uncomplicated acute tubular necrosis show an early, obvious, and persisting nephrogram. This is comparable to the findings of Chamberlain and Sherwood (1967) in experimental animals with acute tubular necrosis. This pattern is seen both in the oliguric and early diuretic recovery phase. In general, but not invariably, oliguria is associated with no pyelogram or only a faint one. Kidney size is normal or possibly increased unless there has been pre-existing renal disease. This nephrographic pattern is not, however, peculiar to patients with acute tubular necrosis as the same pattern was observed in patients with acute renal failure due to severe pyelonephritis. We have not, however, encountered this pattern in any other form of acute renal failure.

These typical urographic findings are modified by associated severe glomerular disease. Thus a delayed, increasingly dense nephrogram was observed when acute tubular necrosis developed on a background of established severe glomerular disease and a very delayed, faint nephrogram was noted in one patient with severe pyelonephritis complicating renal amyloid.

Acute renal failure due primarily to glomerular disease showed a nephrographic pattern quite distinctive from that seen in acute uncomplicated tubular necrosis. Thus, three of the four patients presenting with acute renal failure due to acute glomerulonephritis showed an increasingly dense nephrogram, while the fourth, who had extremely severe glomerular involvement on biopsy and who remained anuric until her death six weeks later, showed no nephrogram up to 24 hours. Patients with chronic glomerulonephritis and malignant essential hypertension showed faint persisting nephrograms. None of the patients with acute golmerulonephritis developed a pyelogram while patients with chronic glomerulonephritis or malignant hypertension, unless anuric, had remarkably good pyelograms. It should be noted that one of the three patients with chronic glomerulonephritis had normal-sized kidneys. This observation serves to emphasize that while chronic glomerulonephritis is usually associated with small contracted kidneys such patients may occasionally present with normal-sized kidneys. This latter finding must therefore not be used as evidence to exclude chronic glomerulonephritis.

In only five of the 32 patients studied was no nephrogram or pyelogram observed. One patient had extremely severe pro- 
liferative glomerulonephritis, two had total kidney infarction, and one had severe interstitial nephritis with, on biopsy, what appeared to be severe tubular compression. The remaining patient had a combination of extensive tubular blockage due to myeloma protein and glomerulonephritis.

The possible mechanisms underlying the development of these various nephrographic patterns have been recently reviewed (Fry and Cattell, 1972). Space precludes any further discussion of these at this time. It must, however, be reemphasized that the diagnostic value of the nephrographic pattern relates to the changes in density observed with time. Precise measurement of absolute density is difficult, depending as it does on many independent variable factors. In general, doubtful changes in density should be ignored and only unequivocal changes recorded. On no account should a diagnosis be attempted when the urographic technique has resulted in unsatisfactory radiographs.

Though based on relatively small numbers of cases these observations confirm that distinctive nephrographic patterns are observed in patients with acute renal failure. They also indicate that more extensive observations are required both to assess more fully the diagnostic value of the nephrographic pattern and to define more clearly the mechanisms underlying the development of the different patterns.

We would like to record our appreciation of the work of the radiographers responsible for these examinations, especially Miss Jill
Baldock, Miss Anne Casey, and Miss Jane Cooper who were the superintendent radiographers. We would also like to thank Mrs. Sylvia Welsh for her invaluable help throughout the study.

\section{References}

Brown, C. B., Glancy, J. J., Fry, I. K., and Cattell, W. R. (1970). Lancet, 2, 952.

Chamberlain, M. J., and Sherwood, T. (1967). Nephron, 4, 65.

Eliahou, H. E. (1964). British Medical fournal, 1, 807.

Eliahou, H. E., and Bata, A. (1965). Nephron, 2, 287.

Fry, I. K., and Cattell, W. R. (1970). British fournal of Hospital Medicine, 3, 67.

Fry, I. K., and Cattell, W. R. (1971a). British fournal of Radiology, 44, 198.

Fry, I. K., and Cattell, W. R. (1971b). British Medical Bulletin, 27, 148.

Fry, I. K., and Cattell, W. R. (1972). British Medical Bulletin, 28, 227.

Graber, I. G., and Sevitt, S. (1959). Fournal of Clinical Pathology, 12, 25.

Levinsky, N. G. (1966). New England fournal of Medicine, 274, 1016.

Luke, R. G., and Kennedy, A. C. (1967). Postgraduate Medical fournal, 43, 280.

Mahaffy, R. G., Matheson, N. A., and Caridis, D. T. (1969). Clinical Radiology, 20, 320.

Meadows, S. R., Cameron, J. S., Ogg, C. S., and Saxton, H. M. (1971). Archives of Disease in Childhood, 46,221 .

Moseley, I. F., McIntosh, C. S., Fry, I. K., and Cattell, W. R. (1971). Proceedings of the European Dialysis and Transplant Association, 8, 537.

Perlmutter, M., Grossman, S. L., Rothenburg, S., and Dobkin, G. (1959). Fournal of the American Medical Association, 170, 1533.

Platts, M. M. (1966). Clinical Science, 30, 453.

Rosenbaum, J. L. (1967). In Renal Failure, ed. A. N. Brest and G. H. Moyer, p. 253. London, Pitman Medical.

Schencker, B. (1964). Radiology, 83, 12.

Wrong, O. M. (1971). British Medical Bulletin, 27, 97.

\title{
Euglycaemic Diabetic Ketoacidosis
}

\author{
J. F. MUNRO, I. W. CAMPBELL, A. C. MCCUISH, L. J. P. DUNCAN
}

British Medical fournal, 1973, 2, 578-580

\section{Summary}

Of a series of 211 episodes of diabetic metabolic decompensation 37 had severe euglycaemic ketoacidosis (a blood sugar level of less than $300 \mathrm{mg} / 100 \mathrm{ml}$ and a plasma bicarbonate of $10 \mathrm{mEq} / 1$. or less). All were young insulindependent diabetics, only one being previously undiagnosed. Vomiting was a common factor, and in all carbohydrate reduction occurred with continued or increased daily insulin dose. Treatment comprised fluid and electrolyte replacement and large doses of insulin covered by adequate carbohydrate, many receiving $10 \%$ dextrose. Alkali was either withheld or given sparingly and the therapy was monitored by serial estimations of plasma bicarbonate. All the patients survived.

\section{Introduction}

During 1964-71 211 episodes of severe diabetic metabolic decompensation were treated in the general medical wards to which the diabetic department is affiliated. In all, either the

Diabetic Department, Royal Infirmary, Edinburgh EH3 9YW

J. F. MUNRO, M.B., F.R.C.P., Senior Registrar (Now Consultant Physician, Eastern General Hospital, Edinburgh)

I. W. CAMPBELL, M.B., M.R.C.P., Pfizer Research Fellow (Now Registrar) A. C. MCCUISH, M.B., M.R.C.P., Registrar (Now Senior Registrar)

L. J. P. DUNCAN, M.B., F.R.C.P., Consultant Physician blood glucose exceeded $650 \mathrm{mg} / 100 \mathrm{ml}$ or the plasma bicarbonate was less than $15 \mathrm{mEq} / 1$. In 11 there was gross hyperglycaemia and hyperosmolarity but no ketoacidosis (Campbell et al., 1973). This paper considers the other extreme of the broad spectrum of diabetic metabolic decompensation-namely, severe ketoacidosis with a plasma bicarbonate of $10 \mathrm{mEq} / \mathrm{l}$. or less without pronounced hyperglycaemia, the blood glucose being less than $300 \mathrm{mg} / 100 \mathrm{ml}$. We have called this syndrome "euglycaemic diabetic ketoacidosis" for lack of a better term.

\section{Details of Patients}

Thirty-seven episodes of euglycaemic diabetic ketoacidosis occurred in 17 patients, 11 of them female. Twelve presented only once, two had two episodes, another two each had three similar admissions, and one required treatment for this condition on 15 occasions. All were young (average age 18.6 years, range 10-28) and with one exception were known insulindependent diabetics (mean duration six years, range two months15 years). The mean daily insulin dose was 101 I.U. (range 26-300) but in only one patient was it greater than 200 I.U. Before 26 episodes occurred satisfactory control had been obtained by the injection each morning of soluble and protamine zinc insulins, in six an additional evening dose of soluble was required, and in four the treatment was Actrapid combined with either Novo Crystal II or insulin zinc suspension (amorphous) injected twice daily. The only previously undiagnosed diabetic gave a one-month history of thirst and for two days before admission had abdominal pain with frequent vomiting. The mean duration of symptoms, of which vomiting was the 